\section{In Vivo Cloning Strategy for Rhizobium Plasmids}

BioTechniques 33:782-788 (October 2002)

\begin{abstract}
We have developed a simple system to clone indigenous Rhizobium plasmids into E. coli. The strategy consists of three matings: the first is to insert Tn5 in the plasmid to be cloned, the second incorporates the integrative vector into the inserted Tn5 in the native Rhizobium plasmid, and the last mating transfers the target plasmid directly into E. coli. This mating-based system was successfully used to clone plasmids of Rhizobium species with sizes ranging from 150 to $270 \mathrm{~kb}$. In addition, a 500-kb fragment of a 600-kb megaplasmid was also cloned. This strategy could be used for cloning indigenous replicons of other Gram-negative bacteria into a different host.
\end{abstract}

\section{INTRODUCTION}

Bacteria of the genus Rhizobium fix nitrogen within nodules that they form in symbiotic association with legumes. Many genes involved in the symbiotic process are encoded on plasmids, whose sizes range from 100 to $1700 \mathrm{~kb}$ (11). Usually, the production of an overlapping collection of cosmids or BAC clones is the first step in generating physical and genetic maps of such large plasmids (18). Unfortunately, the clones obtained are often not large enough and are so random that a large collection of clones is needed to completely cover an entire plasmid. In a previous report (3), we developed a procedure for cloning large fragments from the Sinorhizobium meliloti pExo megaplasmid. Regions cloned were flanked by the RK2 oriT gene in direct orientation. Although amenable to cloning large DNA regions from $S$. meliloti, this method requires a genetic map of the plasmid to isolate the desired regions, a transduction system, and other genetic traits (3).

Here we describe a general system to clone plasmids of Rhizobium species. This in vivo system has several

Table 1. Bacterial Strains and Plasmids Used

\begin{tabular}{|c|c|c|}
\hline $\begin{array}{l}\text { Strains or } \\
\text { Plasmids }\end{array}$ & Relevant Characteristics & $\begin{array}{l}\text { Source or } \\
\text { Reference }\end{array}$ \\
\hline \multicolumn{3}{|l|}{ R. tropici } \\
\hline CFN299 & Wild-type strain Nalr & 14 \\
\hline CFNE68 & pSym::Tn5 mob & This study \\
\hline CFNE84 & pSym::Tn5 mob & This study \\
\hline CFNE87 & pSym::Tn5 mob & This study \\
\hline CFNE130 & CFN299, pcsA::Tn5 mob & 16 \\
\hline CFNE299-19 & CFN299, teu::Tn5 gusA & 20 \\
\hline CFNE299-10 & CFN299, Sym plasmid deleted & 13 \\
\hline \multicolumn{3}{|l|}{ R. etli } \\
\hline CFN42 & Wild-type Nalr & 22 \\
\hline CFNX187 & pCFN42a::Tn5 mob Nalr $\mathrm{Km}^{r}$ & 1 \\
\hline CFN037 & pCFN42b::Tn5 mob Nalr $\mathrm{Km}^{r}$ & 1 \\
\hline CFNX191 & pCFN42c::Tn5 mob Nalr $\mathrm{Km}^{\mathrm{r}}$ & 1 \\
\hline CFNX2001 & $\begin{array}{l}\text { CFN42 cured of pCFN42a } \\
\text { and pCFN42b }\end{array}$ & 10 \\
\hline \multicolumn{3}{|l|}{ S. meliloti } \\
\hline GRM6LR & pRmeGR4a::SSpr/Str & 2 \\
\hline \multicolumn{3}{|l|}{ A. tumefaciens } \\
\hline GMI9023 & Plasmid-less Rifr strain & 19 \\
\hline GMI9023/pCFN42a & pCFN42a::Tn5 mob Kmr Rifr & 6 \\
\hline \multicolumn{3}{|l|}{ E. coli } \\
\hline $\mathrm{DH} 5 \alpha$ & & $\begin{array}{l}\text { Invitrogen (Carlsbad, } \\
\text { CA, USA) }\end{array}$ \\
\hline \multicolumn{3}{|l|}{ Plasmids } \\
\hline pTH509 & $\begin{array}{l}\text { IS50, oriV, } \Omega \mathrm{Sp}^{\mathrm{r}} \text {, oriT, } \mathrm{Cm}^{\mathrm{r}} \\
\text { pBAC F origin }\end{array}$ & 3 \\
\hline pRK2013 & $\begin{array}{l}\text { ColE1 replicon with RK2 transfer } \\
\text { region } \mathrm{Km}^{\mathrm{r}} \mathrm{Nm}^{\mathrm{r}}\end{array}$ & 5 \\
\hline \multicolumn{3}{|c|}{$\begin{array}{l}\text { Sp/St, spectinomycin and streptomycin, respectively. } \\
\text { Rif, rifampicin. }\end{array}$} \\
\hline
\end{tabular}

advantages with respect to the traditional in vitro systems, such as a direct isolation of specific plasmids, fast cloning, and coverage of plasmids or chromosomes with only a few BAC clones. The system is based on three single matings. The principal components of the system are the oriT (RK2) to transfer DNA and the F origin to stably maintain large replicons in $E$. coli (7). Using this system, we were able to clone different plasmids from various Rhizobium species. Our system could be useful in producing large replicons that could then be used for sequencing or evaluating gene expression in different genetic backgrounds.

\section{MATERIALS AND METHODS}

The bacterial strains and plasmids used are listed in Table 1. Rhizobium strains were grown on PY medium (0.5\% peptone, $0.3 \%$ yeast extract, 10 $\mathrm{mM} \mathrm{CaCl}_{2}$ ) supplemented with the following antibiotics: $25 \mu \mathrm{g} / \mathrm{mL}$ kanamycin, $10 \mu \mathrm{g} / \mathrm{mL}$ chloramphenicol, 25 $\mu \mathrm{g} / \mathrm{mL}$ spectinomycin, $30 \mu \mathrm{g} / \mathrm{mL}$ streptomycin, $20 \mu \mathrm{g} / \mathrm{mL}$ rifampicin, and 10 
$\mu \mathrm{g} / \mathrm{mL}$ nalidixic acid. E. coli strains were grown in LB medium $(1 \%$ peptone, $0.5 \%$ yeast extract, and $1 \%$ $\mathrm{NaCl}$ ) supplemented with $25 \mu \mathrm{g} / \mathrm{mL}$ kanamycin, $10 \mu \mathrm{g} / \mathrm{mL}$ chloramphenicol, $25 \mu \mathrm{g} / \mathrm{mL}$ spectinomycin, and 100 $\mu \mathrm{g} / \mathrm{mL}$ ampicillin. E. coli and Rhizobi$u m$ were grown at $30^{\circ} \mathrm{C}$. Conjugation experiments were performed in the presence of pRK2013 as a helper (5) as previously described (4).

Plasmid purification and genomic DNA extraction were performed according to published protocols (21). For hybridization, DNA was purified, digested with BamHI, and transferred from agarose gels to nylon membranes. Probes were labeled with ${ }^{32} \mathrm{P}$ by random primer, and hybridization was carried out under high-stringency conditions (21). Plasmid profiles were obtained by a modified Eckhardt agarose gel electrophoresis method (9). For sequencing, dsDNA was purified with the High Pure plasmid isolation kit (Roche Applied Science, Mannheim, Germany), and sequencing was performed in an automatic

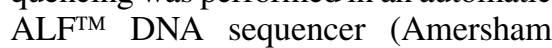
Biosciences, Uppsala, Sweden).

Plant nodulation assays were performed in flasks containing agar as previously described (12). Seeds of Phaseolus vulgaris cv. Negro Jamapa were inoculated with $10^{5}$ Rhizobium cells. Nitrogenase activity was measured by acetylene reduction 21 days after inoculation.

\section{RESULTS AND DISCUSSION}

In general, the technique is as follows. (i) The first mating is carried out with $E$. coli harboring a $\operatorname{Tn} 5$ (23) that is resistant to kanamycin $\left(\mathrm{Km}^{\mathrm{r}}\right)$, while the recipient Rhizobium strain should be resistant to nalidixic acid $\left(\mathrm{Nal}^{\mathrm{r}}\right)$. Rhizobium transconjugants, which are resistant to both kanamycin and nalidixic acid, and thus contain the transposon in the replicon to be cloned, are selected. (ii) In the second mating, these $\mathrm{Km}^{\mathrm{r}}$ $\mathrm{Nal}^{\mathrm{r}}$ Rhizobium transconjugants are mated with $E$. coli harboring the integrative vector pTH509, which contains genes for resistance to chloramphenicol $\left(\mathrm{Cm}^{\mathrm{r}}\right)$ and spectinomycin $\left(\mathrm{Sp}^{\mathrm{r}}\right)$. During this second mating, a single crossover occurs between IS50 of the integrative vector and that of target Tn5 that is inserted in the Rhizobium plasmid. Then, Rhizobium recombinants resistant to kanamycin, chloramphenicol, and nalidixic acid are selected and purified. (iii) In the third mating, Rhizobium transconjugants containing the recombinant cointegrate (i.e., integrative vector pTH509 plus target plasmid) are mated with E. coli DH5 $\alpha$. E. coli exconjugants are selected on LB medium supplemented with chloramphenicol, spectinomycin (integrative vector), and kanamycin (target Rhizobium plasmid). Rhizobium donors are counterselected either by the inability to grow on LB or by a difference in growth rate in comparison to the recipient E. coli. A schematic representation of the cointegrate structure that is generated be- tween the integrative vector pTH509 and the IS50 of the target Rhizobium $\mathrm{Tn} 5$, as well as the resultant plasmid in E. coli, is shown in Figure 1.

We applied our system to clone plasmids of R. etli (22), R. tropici (14), and $S$. meliloti (2). The integrative vector pTH509 was found to insert in the IS50 at a frequency of $10^{-7}$. However, when the Rhizobium plasmid is targeted with Tn5 mob recombination of pTH509 occurred between either IS50 or oriT (data not shown). Transfer of the cointegrate molecules from Rhizobium to $E$. coli occurred at a frequency of $10^{-6}$.

The presence of cointegrated cloned plasmids in E. coli was visualized by Eckhardt gels. Sizes of the cloned replicons ranged from 150 to $270 \mathrm{~kb}$ (Figure $2 A)$. They included pCFN42a (180 kb),

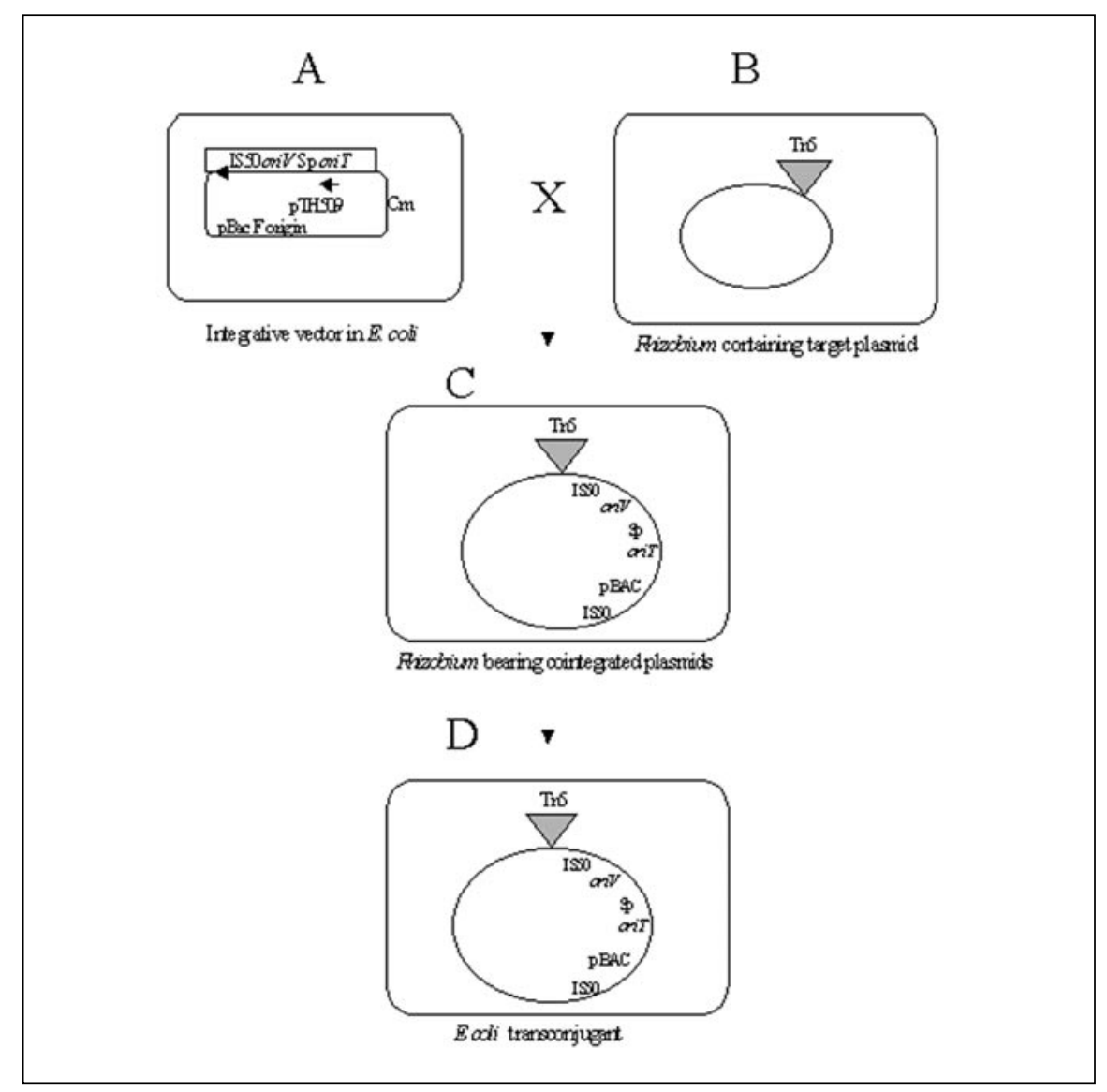

Figure 1. Diagram of the in vivo cloning system. Rhizobium containing a $\mathrm{Tn} 5$ in the target plasmid was mated with E. coli harboring the integrative vector. Rhizobium transconjugants were used as donors for the transfer of the resultant cointegrated molecule in E. coli (see text for details). (A) Schematic representation of the integrative vector pTH509, which contains 296 bp of the IS50 element of Tn5, the oriT of $\mathrm{RK} 2$, the $\mathrm{F}$ origin of replication, and the $\mathrm{Sp}^{\mathrm{r}}$ and $\mathrm{Cm}^{\mathrm{r}}$ cassettes. (B) Schematic representation of the target Rhizobium plasmid labeled with Tn5. (C) The vector pTH509 is integrated into the Rhizobium plasmid by single recombination with the IS50 of Tn5. (D) A third mating between Rhizobium donor and the recipient $E$. coli is necessary to isolate the Rhizobium replicon in E. coli. 
pCFN42 $b(180 \mathrm{~kb})$, and pCFN42c (270 $\mathrm{kb})$ of $R$. etli, as well as pCFN299a (185 kb) of $R$. tropici and pGR4a (150 $\mathrm{kb}$ ) of $S$. meliloti (Figure 2A, lanes 4, 3, 2,6 , and 10, respectively). These replicons were stably maintained in $E$. coli (after 400 generations without antibiotic) and were easily purified by alkaline lysis (21).

To ascertain whether the replicons cloned in E. coli originated from Rhizobium, pCFN42a of $R$. etli rescue from $E$. coli was used as a probe against the $R$. etli CFN42 wild-type strain, $R$. etli CFN42 cured of pCFN42a, Agrobacterium tumefaciens GMI9023 carrying pCFN42 $a$, and A. tumefaciens plasmidless strain GMI9023 (Figure 2B). The results showed that the $R$. etli CFN42 wild-type and $A$. tumefaciens containing pCFN42 $a$ presented the same pattern (Figure 2B, lanes 1 and 4, respectively). $R$. etli devoid of pCFN42 $a$ presented a few weakly hybridizing bands that may result from reiterated sequences elsewhere in the genome (Figure 2B, lane 2 ). In contrast, no hybridization signals were detected in the A. tumefaciens plasmid-less strain (Figure 2B, lane 3). This experiment confirms that $R$. etli pCFN42a was successfully isolated from $E$. coli. Furthermore, the advance in the sequencing project of this replicon revealed that most of the information of pCFN42 $a$ is present in this BAC clone and that the DNA did not suffer major rearrangements during the cloning process (Gonzalez and Davila, unpublished results).

The system is moderately efficient for cloning plasmids of medium size (150-270 kb), since, from 213 clones tested in 18 experiments, $70 \%$ were positives. The remaining 30\% negative clones are found to contain both pTH509 and pRK2013 plasmids. pTH509 resulted by excision from the Rhizobium replicon through homologous re- combination between directed repeats generated during the cointegration event and then was transferred together with pRK2013 in the E. coli recipient strain.

When we tried to clone the $R$. tropici CFN299 symbiotic plasmid (pSym) of approximately $600 \mathrm{~kb}$ (8), only fragments, in the range of $200-500 \mathrm{~kb}$, were obtained (Figure 2A, lanes 7-9, 11, and 12). The regions obtained were overlapping and covered most of the pSym of $R$. tropici CFN299, as tested by PCR and sequence analyses of several genetic markers. For instance, a region containing metabolic genes such as succinate-semialdehyde dehydrogenase, citrate synthase (16), isocitrate lyase, and one insertion sequence (IS) element similar to A. tumefaciens IS3 (17) were identified (data not shown). The presence of these genes on the pSym was also confirmed by hybridization experiments (data not shown). Moreover, to determine the presence of 


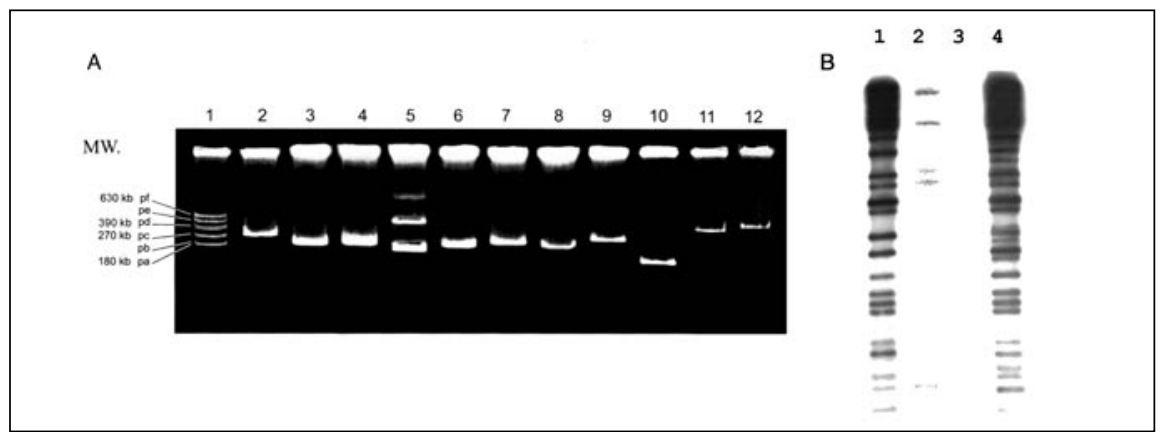

Figure 2. Rhizobium replicons isolated with the three-mating system. (A) Ethidium bromide-stained Eckhardt gel showing Rhizobium replicons cloned in E. coli. Lanes 2, 3, and 4 are plasmid pCFN42c, pCFN42 $b$, and pCFN42 $a$ of $R$. etli, cloned from CFNX191, CFN037, and CFNX187, respectively. Lane 6, pCFN299 $a$ of $R$. tropici obtained from CFNE299-19. Lanes 7-9, 11, and 12 are different regions of the pSym of $R$. tropici isolated from CFNE130, CFNE87, CFNE68, and CFNE84, respectively. Lane 10, plasmid pRmeGR4 $a$ obtained from $S$. meliloti GRM6LR. Lane 1, R. etli CFN42, pa to pf are indigenous plasmids of $R$. etli, and lane 5, R. tropici CFN299, were used as size standards. MW, molecular weight. (B) Autoradiogram of Southern blot of BamHI-digested genomic DNAs, hybridized with the rescue pCFN42a of $R$. etli purified from E. coli. Lane 1, R. etli CFN42; lane 2, R. etli cured of pCFN42a (CFNX2001); lane 3, A. tumefaciens GMI9023 plasmid-less strain; and lane 4, A. tumefaciens GMI9023 containing pCFN42a of R. etli.

nod and fix genes in the isolated CFN299 pSym fragments, complementation experiments were performed. For this purpose, each of the different fragments was transferred to the CFNE29910 strain that has a deletion in the Sym plasmid and is unable to form nodules (13). With this approach, we identified a fragment of $200 \mathrm{~kb}$ on CFNE299-10 that conferred the ability to form nodules and to fix nitrogen in Phaseolus vulgaris. This result suggests that the corresponding region contains genes involved in nodulation and nitrogen fixation. Another isolated fragment appeared to contain the origin of replication of the $R$. tropici CFN299 pSym. This suggestion is based on the fact that this locus is able to autonomously replicate in A. tumefaciens. One reason why we were unable to clone the whole replicon may be that, in Rhizobium cells, deletions are generated by homologous recombination between repeated sequences that are present on the pSym. In previous work (15), $R$. tropici $\mathrm{pSym}$ was shown to contain IS elements that participate in the generation of rearrangements such as amplifications and deletions. Another possible reason for obtaining only fragments of the pSym would be the inability of the $F$ origin to sustain large regions as the entire pSym. However, we were able to obtain clones containing large fragments $(500 \mathrm{~kb})$ that carry most of the information of this replicon.
The cloning procedure presented here is very accessible because few elements are required: the target transposon or cassette in the molecule to be isolated, the integrative vector pTH509 that contains $300 \mathrm{bp}$ of the IS50, an oriV, oriT of RK2, as well as two antibiotic-resistant cassettes $\Omega$ spectinomycin and chloramphenicol. Potentially, all these genetic elements can be used to recombine in the target plasmid. We chose to integrate pTH509 in the $\Omega$ spectinomycin cassette contained in plasmid pRmeGR4a, as well as in the mob site and IS50 element of the transposon Tn 5 mob. The integration of our vector in three different sites and the success of the cloning showed the versatility of our system. In principle, the cloning of plasmids with this method can be applied to a broad range of bacterial species and would be useful to clone gene clusters for subsequent use in the generation of organisms expressing new genetic features.

\section{REFERENCES}

1.Brom, S., A. García de los Santos, T. Stepkowski, M. Flores, G. Davila, D. Romero, and R. Palacios. 1992. Different plasmids of Rhizobium leguminosarum bv phaseoli are required for optimal symbiotic performance. J. Bacteriol. 174:5183-5189.

2.Cebolla, A., F. Ruiz-Berraquero, and J. Palomares. 1993. Stable tagging of Rhizobium meliloti with the firefly luciferase gene for environmental monitoring. Appl. Environ. Micro- 
biol. 59:2511-2519.

3.Chain, P.S., I. Hernández-Lucas, B. Golding, and T.M. Finan. 2000. OriT directed cloning of defined regions from bacterial genomes: identification of the Sinorhizobium meliloti pExomegaplasmid replicator region. J. Bacteriol. 182:5486-5494.

4.Charles, T.C. and T.M. Finan. 1991. Analysis of a 1600-kilobase Rhizobium meliloti megaplasmid using defined deletions generated in vivo. Genetics 127:5-20.

5.Figurski, D.H. and D.R. Helinski. 1979 Replication of an origin-containing derivative of plasmid RK2 dependent on a plasmid function provided in trans. Proc. Natl. Acad. Sci. USA 76:1648-1652.

6.Flores, M., V. González, S. Brom, E. Martínez, D. Piñero, D. Romero, G. Davila, and R. Palacios. 1987. Reiterated DNA sequences in Rhizobium and Agrobacterium spp. J. Bacteriol. 169:5782-5788.

7.Frengen, E., D. Weichenhan, B. Zhao, K. Osoegawa, M. Van Geel, and P.J. de Jong. 1999. A modular, positive selection bacterial artificial chromosome vector with multiple cloning sites. Genomics 58:250-253.

8.Geniaux, E., M. Flores, R. Palacios, and E. Martínez. 1995. Presence of megaplasmids in Rhizobium tropici and further evidence of differences between the two $R$. tropici subtypes. Int. J. Syst. Bacteriol. 45:392-394.

9.Hynes, M.F. and N.F. McGregor. 1990. Two plasmids other than the nodulation plasmid are necessary for formation of nitrogen fixing nodules by Rhizobium leguminosarum. Mol. Microbiol. 4:567-574.

10.Leemans, J., G. Soberón, M.A. Cevallos, L. Fernández, M.A. Pardo, H. De la Vega, M. Flores, C. Quinto, and R. Palacios. 1984. General organization of Rhizobium phaseoli nif plasmids, p. 710. In C. Veeger and V.E. Newton (Eds.). Advances in Nitrogen Fixation Research. Nijhoff, Junk and Pudoc, The Hague, The Netherlands.

11.Martínez, E., D. Romero, and R. Palacios. 1990. The Rhizobium genome. Crit. Rev. Plant Sci. 9:59-93.

12.Martínez, E., M.A. Pardo, R. Palacios, and M.A. Cevallos. 1985. Reiteration of nitrogen fixation gene sequences and specificity of Rhizobium in nodulation and nitrogen fixation in Phaseolus vulgaris. J. Gen. Microbiol. 131:1779-1786.

13.Martínez-Romero, E. and M. Rosenblueth. 1990. Increased bean (Phaseolus vulgaris L.) nodulation competitiveness of genetically modified Rhizobium strains. Appl. Environ. Microbiol. 56:2384-2388.

14.Martínez-Romero, E., L. Segovia, F.M. Mercante, A. Franco, P. Graham, and M.A. Pardo. 1991. Rhizobium tropici a novel species nodulating Phaseolus vulgaris L. beans and Leucaena sp trees. Int. J. Syst. Bacteriol. 41:417-426.

15.Mavingui, P., T. Laeremans, M. Flores, D. Romero, E. Martínez-Romero, and R. Palacios. 1998. Genes essential for nod factor production and nodulation are located on a symbiotic amplicon (AMPRtrCFN299pc60) in Rhizobium tropici. J. Bacteriol. 180:2866-2874.

16.Pardo, M.A., J. Lagúnez, J. Miranda, and E. Martínez. 1994. Nodulating ability of Rhizobi- um tropici is conditioned by a plasmid-encoded citrate synthase. Mol. Microbiol. 11:315-321.

17.Paulus, F., J. Canaday, and L. Otten. 1991. Limited host range Ti plasmids: recent origin from wide host range Ti plasmid and involvement of a novel IS element, IS868. Mol. Plant Microbe Interact. 4:190-197.

18.Perret, X., W. Broughton, and S. Brenner. 1991. Canonical ordered cosmid library of the symbiotic plasmid of Rhizobium species NGR234. Proc. Natl. Acad. Sci. USA 88:19231927.

19.Rosenberg, C. and T. Huguet. 1984. The pAtC58 plasmid of Agrobacterium tumefaciens is not essential for tumor induction. Mol. Gen. Genet. 196:533-536.

20.Rosenblueth, M., M.F. Hynes, and E. Martínez-Romero. 1998. Rhizobium tropici teu genes involved in specific uptake of Phaseolus vulgaris bean-exudate compounds. Mol. Gen. Genet. 258:587-598.

21.Sambrook, J., E.F. Fritsch, and T. Maniatis. 1989. Molecular Cloning: A Laboratory Manual, 2nd ed. CSH Laboratory Press, Cold Spring Harbor, NY.

22.Segovia, L., J.P.W. Young, and E. MartinezRomero. 1993. Reclassification of American Rhizobium leguminosarum biovar phaseoli type I strains as Rhizobium etli sp nov. Int. J. Syst. Bacteriol. 43:374-377.

23.Simon, R. 1984. High frequency mobilization of gram-negative bacterial replicons by the in vitro constructed Tn5-mob transposon. Mol. Gen. Genet. 196:413-420.

We thank J.A. Ramírez-Trujillo, M.A. Gaitan, J.A. Gama, V. González, M.A. Rogel-Hernández, M. Rodriguez, J. Caballero, and M. Dunn for technical help and discussion, as well as Susana Brom who kindly supplied strains. Address correspondence to Ismael Hernández-Lucas, Centro de Investigación sobre Fijación de Nitrógeno, Universidad Nacional Autónoma de México, Apdo. Postal 565-A, Cuernavaca, Morelos, México.e-mail: ismael@cifn.unam.mx

Received 6 May 2002; accepted 16 July 2002.

I. Hernández-Lucas ${ }^{1}$, P. Mavingui ${ }^{1,2}$, T. Finan ${ }^{3}$, P. Chain ${ }^{3}$, and E. Martínez-Romero ${ }^{1}$

${ }^{1}$ Universidad Nacional

Autónoma de México

Cuernavaca, Morelos, México

${ }^{2}$ Université Claude Bernard

\section{Lyon I}

Villeurbanne, France

${ }^{3}$ McMaster University

Hamilton, Ontario, Canada
Yeast tRNA as Carrier in the Isolation of Microscale RNA for Global Amplification and Expression Profiling

BioTechniques 33:788-796 (October 2002)

\section{ABSTRACT}

The characterization of global gene expression patterns of microscale samples is important in many areas of biological and clinical research. The choice of carrier is critical for the efficient isolation and successful amplification of RNA at the nanogram level. Here we show that recovery of nanograms of RNA is significantly higher when carrier linear polyacrylamide is supplemented with carrier tRNA. Reverse transcription and in vitro transcription reactions remain efficient and specific in the presence of carrier tRNA. Finally, comparison of GeneChip ${ }^{T M}$ array hybridization patterns demonstrates that the presence of carrier tRNA does not cause detectable distortion in global amplification. Taken together, tRNA is a superior carrier for the isolation and global amplification of microscale RNA.

\section{INTRODUCTION}

Genome-wide expression profiling using cDNA microarrays or oligonucleotide microarrays $\left(\right.$ GeneChips ${ }^{\mathrm{TM}}$; Affymetrix, Santa Clara, CA, USA) has become an important tool in biomedical studies $(13,18,23)$. Expression data have been obtained from a wide variety of samples including bacteria, fungi, mammalian cells, and tissues $(8,19-22,24)$. The application of array technology generally requires the isolation of RNA from millions of cells containing micrograms to milligrams of total RNA. This is usually achievable only in samples that can be easily propagated in vitro, such as yeast and bacteria. In higher organisms, expression analysis is often crippled by the fact that samples in need of analysis come in only hundreds of cells. 